\title{
0 Pró-PET-Saúde frente aos desafios do processo de formação profissional em saúde
}

Marcelo Viana da Costa(a)

Flávio Adriano Borges ${ }^{(b)}$

Costa MV, Borges FA. The Pro-PET-Health and the challenges of the professional education in health. Interface (Botucatu). 2015; 19 Supl 1:753-63.

This paper aims to explore the major changes induced by the current reorientation policies of health professional education, specifically the National Program for Reorientation of Professional Education in Health and the Education by Work for Health Program (Pro-PETHealth). Reports from these institutions and spokespeople from the Ministry of Health were used as source data, which are available on the FORMSUS platform. We analyzed the data with content analysis. We found that Pro-PET-Health is inducing key changes in health education - for example, strengthening teaching and service, diversifying scenarios for practice and innovation in teaching methods that foster research along with social and healthcare needs, and ultimately stimulating interprofessional education.

Keywords: Health education. Teaching care integration services. Public policies.
Este artigo busca explorar as principais mudanças induzidas pelas atuais políticas de reorientação da formação profissional em saúde, mais especificamente, o Programa Nacional de Reorientação da Formação Profissional em Saúde e o Programa de Educação pelo Trabalho para a Saúde - PróPET Saúde. Foram utilizados como fonte de dados os relatórios das instituições e de assessoria do Ministério da Saúde que se encontram disponibilizados na Plataforma FORMSUS. Foi adotada a análise de conteúdo temática como técnica de análise dos dados. O Pró-PET-Saúde vem induzindo mudanças nevrálgicas na formação em saúde: fortalecimento da articulação ensino/serviço; diversificação dos cenários de práticas e inovação dos métodos de ensino; fomento à pesquisa em articulação com as necessidades sociais e de saúde e estimulando a educação interprofissional.

Palavras-chave: Educação em saúde. Serviços de integração docente-assistencial. Políticas públicas. (a) Departamento de Enfermagem, Campus Avançado Profa. Maria Elisa de Albuquerque Maia, Universidade do Estado do Rio Grande do Norte. BR 405 Km 3, Arizona Pau dos Ferros, RN, Brasil. 59900-000.vianacostam@yahoo.com.br

(b) Doutorando, Programa de PósGraduação em Enfermagem em Saúde Pública, Escola de Enfermagem de Ribeirão Preto, Universidade de São Paulo. Ribeirão Preto, SP, Brasil. flavioborges.enf@gmail.br 


\section{Introdução}

Em anos recentes, países ao redor do mundo têm dedicado importantes esforços na reflexão sobre os desafios que as mudanças atuais da sociedade impõem aos serviços de saúde e, por consequência, ao processo de formação dos profissionais de saúde. O debate tem apontado evidências relevantes que tornam incoerente pensar em mudanças nos serviços de saúde, sem a necessária articulação com processos sólidos e sustentáveis na lógica que fundamenta a formação profissional em saúde ${ }^{1,2}$.

A literatura mais recente, que aborda a problemática da formação dos profissionais e das práticas em saúde, traz como importante referência o relatório elaborado pela Comissão de Educação da Revista Lancet, que se configura como independente e que se debruçou sobre importantes gargalos diante da temática, a partir das perspectivas globais ${ }^{3}$. O escopo desse momento não é esgotar a discussão dos pontos-chave trazidos pelo relatório, mas tê-lo como dispositivo capaz de disparar a reflexão do cenário brasileiro, tendo a clareza do caráter inacabado desse debate.

Entre os desafios apontados pela literatura, estão: a má distribuição dos profissionais de saúde entre diferentes países e no interior de um mesmo território; dificuldade de fixação de profissionais em regiões pobres ou de difícil acesso; pouca aproximação dos profissionais com a realidade de vida e saúde das pessoas; limitada responsabilidade social dos profissionais, acompanhada pela dificuldade na formação do pensamento crítico; formação centrada nas habilidades específicas das categorias profissionais; intensa divisão do trabalho em saúde, forte influência do mercado de trabalho na ordenação dos processos formativos; tendências pedagógicas tradicionais que reproduzem modelos assistenciais também tradicionais, tendo como principal cenário de aprendizagem hospitais ou laboratórios de ensino, entre outros determinantes ${ }^{3,4}$.

Os problemas anteriores são determinados por diversos elementos históricos, sociais, econômicos, culturais, onde se evidencia forte relação de forças de interesses distintos e, por vezes, diversos. Por outro lado, as inúmeras transformações que ocorrem na sociedade têm exigido, cada vez mais, um outro perfil profissional, capaz de efetivar práticas em saúde de forma eficiente e resolutiva, configurando a primeira tensão de forças a ser mencionada ${ }^{5}$.

Por um lado, as necessidades sociais e de saúde, que se manifestam de forma individual e coletiva. Por outro lado, serviços de saúde centrados na oferta de procedimentos. Essa lógica de produção de serviços de saúde procedimento-centrado ou médico-centrado encontra como suporte o processo de formação que apresenta as características anteriormente citadas, e que, numa permanente articulação, desenha um cenário de enfrentamento difícil e complexo $0^{6,7}$.

Pensando neste cenário, é possível considerar como fio condutor, que provoca outras análises, a forte tendência de formar profissionais de saúde em suas habilidades e competências específicas, constituindo o que é definido como silos profissionais. Para essa realidade, podem ser atribuídos, entre outros elementos, as identidades profissionais definidas e legitimadas pela sociedade, agregando, às profissões, status e relevância social diferenciadas ${ }^{8}$.

Ainda como desdobramento desse novelo e como legado da perspectiva produtivista da sociedade capitalista, a divisão do trabalho em saúde acentuou os problemas já existentes. A divisão do trabalho se reproduzia no processo de formação, sendo o contrário uma verdade. O trabalho dividido é a herança de toda essa problemática. As categorias profissionais não se veem como parte do trabalho coletivo em saúde, mas como um mundo à parte deste 9 .

O usuário - e suas necessidades - assume papel de mero objeto de intervenção, e o trabalho em equipe passa a não ter sentido numa lógica em que cada profissional faz o que lhe compete, sem a preocupação de (re)significar as relações estabelecidas na produção dos serviços de saúde. A colaboração, o agir comunicativo, a interação passam a não fazer parte da dinâmica do processo de trabalho em saúde, com forte agravante para todo o Sistema de Saúde ${ }^{10,11}$.

As problemáticas apresentadas, por sua vez, demandam um processo de enfrentamento articulado e a formação profissional em saúde possui papel determinante neste processo. Neste sentido, este artigo tem como objetivo explorar as principais mudanças induzidas pelas atuais políticas de reorientação da formação profissional em saúde, mais especificamente, o Programa Nacional de Reorientação da Formação Profissional em Saúde e o Programa de Educação pelo Trabalho para a 
Saúde - Pró-PET-Saúde, a partir dos relatórios das instituições e de assessoria do Ministério da Saúde e à luz da literatura científica.

\section{Pró-PET-Saúde: importantes acúmulos históricos na reorientação do processo de formação dos profissionais de saúde}

A preocupação com o processo de formação dos profissionais em saúde no Brasil não é recente. $O$ país acompanhou o movimento global com o escopo de adequar os perfis profissionais às necessidades de saúde da população e ao fortalecimento dos Sistemas Nacionais de Saúde ${ }^{12,13}$. Assim, a discussão em torno da elaboração de propostas que pudessem se configurar como contra-hegemônicas, antecede até mesmo a criação e implantação do Sistema Único de Saúde (SUS), sendo contemporânea do movimento da medicina comunitária, que ganhou força no âmbito dos departamentos de medicina preventiva. $\mathrm{O}$ processo de formação ganha destaque nesse momento histórico, à medida que se discutia a incoerência de uma reforma do setor saúde, sem a necessária reforma do processo de formação da força de trabalho, que seria a sustentação deste ${ }^{14}$.

O processo de formação dos profissionais de saúde começa a ocupar espaço nas conferências nacionais de saúde, sendo um dos temas incluídos na proposta da Reforma Sanitária Brasileira. Deste período até a atualidade, as iniciativas, movimentos e políticas com foco na reorientação da formação em saúde assumiram configurações distintas, muitas vezes, mudando o tom por força das concepções de grupos. Essa realidade, presente na literatura nacional, mostra a fragilidade, em alguns momentos da história, das iniciativas enquanto política de Estado, à medida que transitava de formato à mudanças de governo ${ }^{15}$.

Porém, o propósito não é explorar à exaustão todas as iniciativas/políticas que induziram importantes reflexões e mudanças sobre a formação dos profissionais de saúde no Brasil. Todas as políticas foram relevantes para acumular experiências no sentido de, cada vez mais, se pensar na viabilidade de vários processos de mudanças coerentes com as necessidades de transformações na lógica dos serviços de saúde, e, consequentemente, na vida e saúde das pessoas.

Nesse sentido, será dada centralidade às políticas mais recentes, sem desconsiderar, e nem é possível, o acúmulo histórico das conquistas viabilizadas em momentos anteriores. Assim, serão trazidos aspectos do Programa de Reorientação da Formação Profissional em Saúde - Pró- Saúde, e o Programa de Educação pelo Trabalho para a Saúde - PET-Saúde, para esta explanação.

As atuais políticas de estímulo às mudanças na formação em saúde tomam como instrumento orientador as Diretrizes Curriculares Nacionais (DCN) que, por sua vez, representaram um grande avanço nos movimentos para reorientarem a formação dos profissionais de saúde, por atentarem para a necessidade de se formar um profissional humanista, com competências não apenas técnica, mas, também, ética e política; pela intenção de se formar um profissional de saúde apto a assumir a responsabilidade frente à consolidação do SUS; formar a capacidade crítica e reflexiva dos profissionais, para que os mesmos possam identificar as necessidades de saúde de uma forma mais ampliada, e pela ênfase dada à integralidade da atenção, valorizando todos os níveis de atenção, assim como todas as categorias profissionais, dando centralidade às necessidade do usuários, expressas nas dimensões individuais e pedagógicas ${ }^{16,17}$.

É claro que a efetivação dessas diretrizes varia muito em decorrência das diferentes visões de mundo e da identificação com as bases ideológicas que fundamentaram a formulação das DCN, mas, sem dúvida, é uma ferramenta indispensável na superação de um dos maiores entraves para o SUS: a formação de seus profissionais ${ }^{16,18}$. Nesse sentido, outras políticas começam a ser elaboradas para que as limitações das iniciativas anteriores possam ser superadas, efetivando sucessivos processos de avaliação e discussão do tema.

Dentre as políticas mais recentes, merece destaque o Pró-Saúde - Programa de Reorientação da Formação Profissional em Saúde, instituído pela portaria 2.101 de 3 de novembro de 2005. A primeira chamada do Pró- Saúde envolveu apenas os cursos de medicina, enfermagem e odontologia, por serem as categorias profissionais que integram as equipes de referência na Estratégia Saúde da Família. Porém, a portaria 3.019 de 26 de novembro de 2007 amplia o Pró-Saúde para todos os cursos da área da saúde. 
A política se configura como relevante ferramenta de incentivo à superação do tradicional modelo de formação em saúde, dando importância aos processos sociais e econômicos da população, a partir da compreensão ampliada de saúde; fortalecimento da articulação com os serviços públicos de saúde, fortalecendo as ações de prevenção e promoção da saúde; reconfiguração da pesquisa, com foco na produção do conhecimento voltado para o fortalecimento do sistema de saúde; adoção de novas estratégias pedagógicas e da atenção básica como importante espaço de formação ${ }^{19,20}$.

O Pró-Saúde se fundamenta em três grandes eixos: orientação teórica com foco no fortalecimento da compreensão dos determinantes sociais do processo saúde-doença. Este eixo tem como escopo, valorizar os perfis epidemiológicos e as intervenções com vistas à transformação destes; produzir conhecimento coerente com as demandas apontadas pela realidade de vida e saúde da população, bem como pelas necessidades de fortalecimento do SUS; e viabilizar a pós-graduação e educação permanente para que os processos de educação e formaçãa estejam atrelados às necessidades de fortalecimento de áreas estratégicas do SUS ${ }^{19,21}$.

Outro eixo é o Cenário de Práticas que objetiva fortalecer a articulação ensino/serviço, diversificação dos cenários de práticas, de forma que a realidade sirva como espaço de formação, reflexão e integração dos serviços próprios das instituições formadoras com o SUS. Esse eixo fortalece a ideia de articulação e entende a grande relevância da realidade dos serviços para a formação do perfil profissional adequado para o enfrentamento dos problemas apresentados nas realidades ${ }^{19}$.

O último eixo é a orientação pedagógica que se debruça sobre as mudanças curriculares e das práticas pedagógicas que fundamentam o processo de ensino-aprendizagem. Esse eixo fundamenta a construção do perfil crítico e reflexivo, valorizando estratégias ativas de aprendizagem, colocando o aluno como sujeito na construção do conhecimento, problematizando a realidade, amparado na lógica da ação-reflexão-ação. Como propostas para a mudança nos desenhos curriculares, é sugerido que a atenção seja inserida como importante espaço de formação, para dar suporte à lógica de formação do perfil generalista ${ }^{19}$.

Porém, outras iniciativas, como desdobramento do Pró-Saúde, asseguram oportunidades de aproximação entre diferentes atores das diversas profissões, a exemplo do Programa de Educação pelo Trabalho em Saúde (PET-Saúde) ${ }^{22}$. O programa é produto das avaliações das estratégias adotadas no Pró-Saúde e entendido como ferramenta de seu fortalecimento. A proposta tem como eixo condutor a indissociabilidade entre ensino, pesquisa e extensão, e deixa mais clara a intenção de adotar a interdisciplinaridade como estratégia para experienciar a realidade como um processo de formação 20,23 .

Outra inovação proposta pelo PET-Saúde foi a introdução da pesquisa como estratégia de formação e reconhecimento das necessidades dos serviços de saúde e da população. Nesse intuito, tem ampliado as ações de pesquisa clínica, pesquisa-ação e pesquisa sobre o ensino-aprendizagem e gestão. Esta iniciativa permite a permanente articulação entre os processos de formação e a realidade do SUS, tanto em nível de graduação como de pós-graduação ${ }^{23-25}$.

A iniciativa tem como pretensão o estímulo a novos métodos de ensino-aprendizagem, à medida que adota o ensino tutorial e permite a aproximação, nos espaços de produção dos serviços de saúde da atenção básica, de alunos e professores de diferentes cursos da área da saúde ${ }^{26}$. No entanto, ainda se faz necessária a ampliação das pesquisas em torno do PET-Saúde como forma de entender e avaliar como se materializa a articulação e interação entre esses diferentes personagens e suas contribuições para o trabalho colaborativo ${ }^{24}$.

\section{Metodologia}

Pela natureza do trabalho e do objetivo desta pesquisa, optou-se por um estudo exploratório de abordagem qualitativa.

O estudo tem como objeto de investigação dados secundários obtidos pela consulta aos relatórios produzidos pelas Instituições de Ensino Superior - IES que participam do Pró-PET-Saúde: relatório técnico e de visitas da equipe assessora do Ministério da Saúde. O acesso a estes relatórios foi possível por meio da plataforma FORMSUS. 
Foram pesquisados cento e vinte relatórios técnicos anuais do Pró- Saúde e PET-Saúde/Redes de Atenção e 99 relatórios de visita do Pró-PET-Saúde. Os relatórios técnicos anuais são preenchidos pelas coordenações dos projetos na IES e trazem, de forma descritiva, as repercussões do Pró-PET-Saúde em diversos âmbitos do processo de formação em saúde. Os relatórios de visita são preenchidos pela equipe assessora do Ministério da Saúde que acompanha a execução dos projetos.

Os dados obtidos foram analisados a partir da análise de conteúdo temática categorial, observando: as etapas de leitura flutuante, constituição do corpus da pesquisa, elaboração de hipóteses e objetivos $^{27,28}$.

A leitura dos relatórios permitiu categorizar os resultados em quatro principais temas, que coincidem com os objetivos das duas políticas: fortalecimento da articulação ensino-serviço como estratégia de diversificação dos cenários de práticas e fortalecimento da atenção primária, mudanças curriculares e adoção de métodos de ensino e aprendizagem ativos, educação interprofissional como estratégia para qualificar os profissionais de saúde para o trabalho em saúde, e reorientação da pesquisa no âmbito das mudanças no processo de formação.

\section{Resultados}

O processo de categorização dos dados permitiu construir unidades de análises muito semelhantes aos objetivos das políticas. Embora estes objetivos já sejam conhecidos e, por sua vez, espera-se que naturalmente sejam alcançados, a leitura dos relatórios aponta que alguns deles são relatados de forma mais contundente, apontando para o entendimento de que são os principais impactos da política. Outros aparecem com menos frequência, fornecendo elementos de reflexões relevantes.

A articulação ensino-serviço é, sem dúvida, o principal ponto forte trazido nos relatórios. As IES direcionam para o fato de que o Pró-PET-Saúde induziu os cursos a adotarem a articulação ensinoserviço como estratégia de reorientação da formação em saúde. Essa articulação tem permitido o desencadeamento de um processo de inserção do aluno na realidade de vida e saúde da população e da produção dos serviços de saúde, com relevantes ganhos para a formação de um profissional que conheça as demandas que emergem destes cenários.

"O maior avanço foi o da adequação das práticas da comunidade com os conteúdos dos módulos curriculares e a construção de instrumento de avaliação em cenários reais na APS". (IES 2)

“O Pró-Saúde e PET-Saúde fortalecem a integração Ensino - Serviço - Comunidade e incentivam a ampliação dos cenários de prática na medida em que docentes e discentes dos cursos envolvidos e profissionais de saúde trabalham integrados em disciplinas afins e nos projetos". (IES 5)

"A articulação dos cursos de graduação com o Pró-Saúde e PET-Saúde vem se materializando e dando visibilidade à integração ensino- serviço-comunidade como uma estratégia de mudança na formação em saúde". (IES 12)

É interessante relatar que as mudanças expressas no relatório acontecem de forma muito articulada, sendo possível identificar os impactos da política em diversos aspectos da formação. O fortalecimento da articulação ensino-serviço impulsionou a adoção de novas estratégias metodológicas, bem como de mudanças nos desenhos curriculares.

Cursos da área da saúde que historicamente mantinham relação mais distanciada dos serviços passaram por algumas mudanças curriculares para assegurar coerência com as diretrizes do Pró-PETSaúde, incorporando mudanças significativas no processo de formação. 
“Desde a implantação do Pró-Saúde e PET-Saúde o curso de Nutrição propôs um novo Projeto Pedagógico, com inserção precoce dos estudantes nos cenários de prática no SUS e na sua área de territorialização, com o fortalecimento dos componentes orientação teórica, pedagógica e cenários de prática". (IES 31)

"As iniciativas de integração ensino-serviço no período que antecedeu a participação do curso de Farmácia no programa foram pontuais e sem sustentação. A inserção do curso no programa Pró-Saúde e PET-Saúde motivaram mudanças que foram desde a reorientação de algumas disciplinas à contratação de mais docentes com o perfil para a saúde pública e APS". (IES 19)

“Os projetos pedagógicos atuais de todos os cursos já contemplam disciplinas curriculares obrigatórias baseadas na comunidade, reforçadas pelo edital Pró- Saúde II, PET Saúde/ Saúde da Família e Saúde Mental. Com as mudanças curriculares, estes passaram a ampliar a diversificação dos cenários de prática e as redes sociais de apoio". (IES 43)

Outro aspecto identificado nos relatórios é a interação entre os diversos cursos da área da saúde, estimulada pelo Pró-PET-Saúde. As mudanças curriculares com adoção de novas estratégias metodológicas de ensino-aprendizagem têm estimulado a aproximação destes cursos. Embora, nos relatos, se perceba certa confusão conceitual, sobretudo, com relação à interprofissionalidade, fica clara a intenção de viabilizar momentos compartilhados de aprendizagem, com importantes ganhos para a mudança da lógica da educação profissional em saúde.

"Além disso tem promovido eventos para discutir a integração dos cursos e as tendências de mudança na para da saúde, com destaque para o Primeiro Fórum de Ensino da Saúde. O PróPET-Saúde tem desencadeado forte movimento de articulação e integração dos cursos da área da saúde e outros". (IES 7)

"Implantação de módulo integrado interprofissional; Modificação de autopercepção dos cursos como integrantes da área da saúde (educação física)". (IES 12)

“Criação de uma Disciplina Complementar de Graduação (DCG) intitulada Formação Profissional Interdisciplinar para o SUS, ofertada para os acadêmicos dos diferentes cursos da área da saúde. O PET-Saúde permitiu a atuação multiprofissional com integração de diferentes cursos". (IES 21)

Por fim, outra frente de mudanças está no desenvolvimento de pesquisas a partir das experiências vivenciadas pelos alunos nos diversos cenários e estratégias de aprendizagem. A pesquisa assume papel relevante nesse processo de mudanças por estimular os atores envolvidos a produzirem conhecimentos capazes de refletir e encontrar respostas, ou estratégias de enfrentamento, para os problemas de vida e saúde das pessoas, estimulando a capacidade de crítica, reflexão e responsabilidade social.

“Os temas de pesquisa foram delineados a partir de discussões coletivas entre tutores, preceptores e bolsistas inseridos nos grandes projetos. Para tanto, foram acolhidas sugestões de outros profissionais dos cenários de prática, não inseridos diretamente na proposta do Pró-PETSaúde". (IES 16)

"Todos os projetos de pesquisa selecionados foram construídos a partir da identificação de necessidades de saúde pelos preceptores e alunos, com a supervisão acadêmica dos tutores". (IES 17) 
"Os temas de pesquisa apresentam correlação com demandas geradas no âmbito das redes de atenção à saúde do Município [...], tendo sido definidos de forma coletiva. Houve participação dos profissionais na identificação/concepção do problema de pesquisa? Sim, os dois primeiros seminários Pró-Saúde e PET-Saúde foram realizados para agregar potenciais ideias e propostas para subsidiar a elaboração dos projetos de pesquisas, contanto com a participação de: tutores, preceptores, alunos bolsistas e voluntários". (IES 47)

Os relatórios têm apontado, com riqueza de detalhes, a potência do Pró-PET-Saúde frente aos desafios atuais do processo de formação dos profissionais de saúde. São mudanças relevantes e que repercutem em toda a dinâmica do processo. As IES também relatam problemas ou dificuldades na execução de um novo projeto de formação, mas que se configura como algo esperado, frente à histórica tendência da formação tradicional.

Porém, os projetos têm demonstrado boa capacidade de enfrentamento destes problemas e apresentam-se robustos e sustentáveis. Essa sustentabilidade está assegurada pela forte relação com os serviços de saúde, à medida que os processos vêm se complementando de forma natural, criando laços significativos para a formação, para os serviços e gestores.

\section{Discussão}

Os dados apresentados apontam para relevantes avanços no processo de formação profissional na área da saúde. Como mencionado anteriormente, o Pró-PET-Saúde é resultado de diversos esforços históricos no sentido de assegurar novos perfis profissionais mais coerentes com as demandas que emergem no contexto social. Seus princípios e diretrizes são frutos de um contínuo amadurecimento de outras políticas, num processo permanente de avaliação e melhoria constante para aperfeiçoar as ações em torno da formação em saúde ${ }^{20,26}$.

Desta forma, vê-se necessária a ênfase dada ao processo de fortalecimento da articulação ensinoserviço, marcadamente presente nos relatórios do Pró-PET-Saúde. Essa articulação vem sendo desejada desde as primeiras iniciativas de reorientação da formação profissional em saúde, por entender que os profissionais de saúde precisam vivenciar o contexto sociocultural e a dinâmica de produção dos serviços de saúde como uma condição para uma formação profissional de qualidade.

A vivência da dinâmica da produção dos serviços de saúde, no processo de formação, assegura uma formação mais ampliada na medida em que os atores envolvidos são estimulados a orientarem os processos de ensino e aprendizagem pelas demandas visualizadas na realidade prática. Docentes, alunos, preceptores, gestores e usuários se veem compelidos a adotarem uma nova perspectiva de educação muito próxima das necessidades sociais e de saúde ${ }^{26}$.

Esse aspecto contempla um dos grandes problemas identificados na formação em saúde: o pouco conhecimento das realidades, que é consequência de uma formação com forte apelo tecnicista e isolacionista. Produções teóricas têm trazido, com forte ênfase, a necessidade de se qualificarem os estudantes para realizarem análises mais amplas das condições de saúde, de vida e da dinâmica dos sistemas e serviços ${ }^{3}$.

O processo de articulação do ensino ao serviço, incentivado pelo Pró-PET-Saúde, pode ser identificado como a principal característica e ganho desta estratégia política. Não apenas pela adoção de novos cenários de prática, ou de novas estratégias mais ativas e dinâmicas de ensino e aprendizagem, mas pela capacidade de estimular o pensamento crítico, a reflexão e a responsabilidade social diante dos problemas e experiências vivenciadas ${ }^{29}$.

Um dos principais desafios da formação profissional de saúde está exatamente na necessidade de formar sujeitos comprometidos com o fortalecimento do SUS e com a melhoria de vida e saúde de todas as pessoas. Para tanto, superar a lógica de formação centrada, exclusivamente, nas competências e habilidades específicas, sem a necessária formação da competência politica e ética, deve ser a lógica do processo de mudança da educação dos profissionais de saúde ${ }^{30}$. 
Nesse sentido, é possível afirmar que o Pró-PET-Saúde vem cumprindo seu papel quando diversifica os cenários de aprendizagem, dando oportunidade à formação de outras habilidades e competências importantes ao profissional de saúde. Da mesma forma, a adoção de metodologias ativas tem tido enorme relevância no conjunto das mudanças implantadas, à medida que as perspectivas tradicionais não conseguem manter coerência com um grande projeto de mudanças na área de formação dos profissionais de saúde ${ }^{31}$.

Também é necessário destacar que, no conjunto de mudanças estimuladas pelo Pró-PET-Saúde, a educação interprofissional vem ganhando visibilidade quando se percebe a necessidade de estimular, ainda no processo de formação, habilidades e valores que deem sustentação ao trabalho colaborativo nas equipes de saúde.

Experiências demonstram que alunos que vivenciam processos de formação compartilhados apresentam maior interesse e abertura para trabalhar em equipe, entendendo sua função e relevância, bem como dos demais sujeitos envolvidos no trabalho em saúde ${ }^{32}$. A educação interprofissional é potente na superação dos estereótipos e na redução das relações hostis impostas pela cultura da hierarquia nas equipes de saúde $\mathrm{e}^{33-35}$.

Outro ganho da adoção da educação interprofissional no contexto das atuais políticas é o resgate do lugar dos usuários, e suas necessidades, na centralidade do processo de produção dos serviços de saúde $^{36}$. Nessa perspectiva, se fortalecem os processos de mudança da lógica assistencial pelo resgate da perspectiva do trabalho coletivo em saúde, base de sustentação da integralidade da atenção.

A pesquisa, por sua vez, se configura como um potente instrumento dessas mudanças. A produção do conhecimento se aproxima da realidade dos serviços e da vida das pessoas, elaborando produções que são capazes de iluminar mudanças na realidade destes serviços de saúde ${ }^{37}$. Além disso, a pesquisa estimulada no âmbito do Pró-PET-Saúde possui uma outra característica que merece destaque, que é a de aproximar os profissionais de saúde dos alunos, retomando um ritmo de qualificação importante para a melhoria da qualidade da atenção à saúde ${ }^{38}$.

\section{Considerações finais}

As mudanças no processo de formação profissional em saúde se constituem como processos complexos e dinâmicos, assim como as necessidades sociais e de saúde. Nesse sentido, é coerente afirmar que o Pró-PET-Saúde vem exercendo papel determinante na indução de mudanças significativas no âmbito das IES.

O estudo demonstrou que os desafios para a efetiva implementação dos objetivos propostos pelos projetos em questão vêm sendo enfrentados de forma muito sólida e sustentável pelas atuais políticas, à medida que instalam uma nova lógica de formação que envolve serviços de saúde e instituições formadoras.

Embora esteja muito evidente a força do Pró-PET-Saúde no fortalecimento da articulação ensinoserviço, é verdade que esse aspecto tem provocado outras mudanças relevantes, tais como: a forma de ensinar e aprender, de se relacionar e refletir sobre os problemas de saúde, além da interação com os usuários e com os atores das demais categorias profissionais.

É evidente a necessidade de fortalecer os ganhos impulsionados pelo Pró-PET-Saúde, bem como de se aproximar das limitações ou dificuldades na execução dos projetos. Os relatórios trazem, com riqueza de detalhes, a forte articulação das políticas atuais com o debate cotidiano que envolve a educação dos profissionais de saúde. Não se podem perder de vista esses resultados, pela capacidade de provocarem mudanças na realidade dos serviços e da Educação Superior.

Contudo, o atual artigo não tem a pretensão de esgotar essa discussão a partir dos resultados e das análises aqui expostos. Pretende-se desencadear novos processos e novas análises por meio da problematização em torno da configuração das políticas públicas indutoras de uma formação mais coerente com a prática profissional em saúde, tendo como perspectivas, por exemplo: estudos sensíveis à identificação de como tem sido, para os alunos e IES, a implementação deste processo contra-hegemônico de formação em saúde. 


\section{Colaboradores}

Marcelo Viana da Costa participou de todas as etapas da elaboração do artigo (idealização, confecção, padronização e submissão). Flávio Adriano Borges participou das etapas de confecção, padronização, e auxiliou no processo de submissão do artigo, segundo as normas padronizadas pela revista.

\section{Referências}

1. Stiqler FL, Duvivier RJ, Weggemans $M$, Salzer HFJ. Health professionals for the 21st century: a students' view. Lancet. 2010;376(9756):1877-8. http://dx.doi.org/10.1016/ S0140-6736(10)61968-X

2. Horton R. A new epoch for health professionals' education. Lancet. 2010;376(9756):1875-7. http://dx.doi.org/10.1016/S0140-6736(10)62008-9

3. Frenk J, Chen L, Bhutta ZA, Cohen J, Crisp N, Evans T, et al. Health professionals for a new century: transforming education to strengthen health systems in an interdependent world. Lancet. 2010;376(9756):1923-58. http://dx.doi.org/10.1016/S01406736(10)61854-5

4. Boelen C, Dharamsi S, Gibbs T. The social accountability of medical schools and its indicators. Educ Health. 2012;25(3):180-94. http://dx.doi.org/10.4103/13576283.109785

5. Barbosa MA, Brasil VV, Sousa ALL, Monego ET. Refletindo sobre o desafio da formação do profissional de saúde. Rev Bras Enferm. 2003;56(5):574-6. http://dx.doi.org/10.1590/ S0034-71672003000500022

6. Feuerwerker LCM, Cecílio LCO. O hospital e a formação em saúde: desafios atuais. Ciênc Saúde Coletiva. 2007;12(4):965-71. http://dx.doi.org/10.1590/S141381232007000400018

7. Amâncio Filho A. Dilemas e desafios da formação profissional em saúde. Interface (Botucatu). 2004;8(15):375-80. http://dx.doi.org/10.1590/S1414-32832004000200019

8. Kreindler SA, Dowd DA, Dana Star N, Gottschalk T. Silos and social identity: the social identity approach as a framework for understanding and overcoming divisions in health care. Milbank Q. 2012;90(2):347-74. http://dx.doi.org/10.1111/j.14680009.2012.00666.x

9. Matos E, Pires DEP, Sousa GW. Relações de trabalho em equipes interdisciplinares: contribuições para novas formas de organização do trabalho em saúde. Rev Bras Enferm. 2010;63(5):775-81. http://dx.doi.org/10.1590/S0034-71672010000500013

10. Weller J, Boyd M, Cumin D. Teams, tribes and patient safety: overcoming barriers to effective teamwork in healthcare. Postgrad Med J. 2014; 90(1061):149-54. http://dx.doi. org/10.1136/postgradmedj-2012-131168

11. Peduzzi M. Trabalho e educação na saúde: ampliação da abordagem de recursos humanos. Ciênc Saúde Coletiva. 2013;18(6):1539-41. http://dx.doi.org/10.1590/S141381232013000600005

12. Feuerwerker LCM. Educação na saúde: educação dos profissionais de saúde - um campo de saber e de práticas sociais em construção. Rev Bras Educ Med. 2007; 31(1):3-4. http://dx.doi.org/10.1590/S0100-55022007000100001

13. Batista CB. Movimentos de reorientação da formação em saúde e as iniciativas ministeriais para as universidades. Barbaroi. 2013;38:97-125.

14. González AD, Almeida MJ. Movimentos de mudança na formação em saúde: da medicina comunitária às diretrizes curriculares. Physis. 2010;20(2):551-70. http://dx.doi. org/10.1590/S0103-73312010000200012 
15. Dias HSA, Lima LD, Teixeira M. A trajetória da política nacional de reorientação da formação profissional em saúde no SUS. Ciênc Saúde Coletiva. 2013;8(6):1613-24. http://dx.doi.org/10.1590/S1413-81232013000600013

16. Rossoni E, Lampert J. Formação de profissionais para o Sistema Único de Saúde e as diretrizes curriculares. Bol Saúde. 2004;18(1):87-98.

17. Pereira IDF, Lages I. Diretrizes curriculares para a formação de profissionais de saúde: competências ou práxis? Trab Educ Saúde. 2013;11(2):319-38. http://dx.doi. org/10.1590/S1981-77462013000200004

18. Ceccim RB, Carvalho YM. Formação e educação em saúde: aprendizados com a saúde coletiva. In: Campos GWS, Minayo MCS, Akerman M, Drumond Jr. M, Carvalho YM, org. Tratado de saúde coletiva. São Paulo: Hucitec; Rio de Janeiro: Fiocruz; 2006. p. 149-82.

19. Ministério da Saúde (BR); Ministério da Educação (BR). Programa Nacional de Reorientação da Formação Profissional em Saúde - Pró-Saúde: objetivos, implementação e desenvolvimento potencial. Brasília, DF: Ministério da Saúde; 2007.

20. Haddad AE, Morita MC, Pierantoni CR, Brenelli SL, Passarella T, Campos FE. Formação de profissionais de saúde no Brasil: uma análise no período de 1991 a 2008. Rev Saúde Pública. 2010;44(3):383-91. http://dx.doi.org/10.1590/S0034-89102010005000015

21. Ministério da Saúde (BR); Ministério da Educação (BR). Portaria Interministerial $\mathrm{n}^{\circ} 2.101$, de 3 de novembro de 2005. Dispõe sobre os profissionais que compõem a Comissão Executiva do Pró-Saúde. Diário Oficial União. 4 nov 2005;seção 2:25.

22. Ministério da Saúde (BR); Ministério da Educação (BR). Portaria Interministerial $n^{\circ}$ 1.802, de 26 de agosto de 2008. Institui o Programa de Educação pelo Trabalho para a Saúde-PET-Saúde. Diário Oficial União. 26 ago 2008;Seção 1:97.

23. Tanaka EE, Ortiz DA, Neves G, Penteado MM, Dezan CC, Codato LAB, et al. Projeto PET-Saúde: ferramenta de aprendizado na formação profissional em saúde. Rev Bras Educ Med. 2012;36(1 Supl. 2):136-40. http://dx.doi.org/10.1590/S010055022012000300019

24. Ferraz L. O PET-Saúde e sua interlocução com o Pró-Saúde a partir da pesquisa: o relato dessa experiência. Rev Bras Educ Med. 2012; 36(1 Supl. 1):166-71. http://dx.doi. org/10.1590/S0100-55022012000200023

25. Canônico RP, Brêtas ACP. Significado do Programa Vivência e Estágios na realidade do Sistema Único de Saúde para formação profissional na área. Acta Paul Enferm. 2008;21(2):256-61. http://dx.doi.org/10.1590/S0103-21002008000200004

26. Haddad AE, Brenelli SL, Cury GC, Puccini RF, Martins MA, Ferreira JR et al. Pró-Saúde e PET-Saúde: a construção da política brasileira de reorientação da formação profissional em saúde. Rev Bras Educ Med. 2012;36(1 Supl. 1):3-4. http://dx.doi.org/10.1590/ S0100-55022012000200001

27. Minayo MCS. O desafio do conhecimento: pesquisa qualitativa em saúde. 11a ed. Rio de Janeiro: Hucitec; 2008.

28. Bardin L. Análise de conteúdo. Lisboa: Edições 70; 2009.

29. Boelen C, Woollard B. Social accountability and accreditation: a new frontier for educational institutions. Med Educ. 2009;43(9):887-94. http://dx.doi.org/10.1111/ j.1365-2923.2009.03413.x

30. Crisp N, Chen L. Global supply of health professionals. N Engl J Med. 2014;370(10):950-7. http://dx.doi.org/10.1056/NEJMra1111610

31. Silva MAM, Amaral JHL, Senna MIB, Ferreira EF. O Pró-Saúde e o incentivo à inclusão de espaços diferenciados de aprendizagem nos cursos de odontologia no Brasil. Interface (Botucatu). 2012;16(42):707-17. http://dx.doi.org/10.1590/S141432832012000300010 
32. Freitas PH, Colome JS, Carpes AD, Backes DS, Beck CLC. Repercussões do PET-Saúde na formação de estudantes da área da Saúde. Esc Anna Nery. 2013;17(3):496-504. http:// dx.doi.org/10.1590/S1414-81452013000300013

33. DeMatteo DJ, Reeves S. Introducing first year students to interprofessionalism: exploring professional identity in the "enterprise culture": a Foucauldian analysis. J Interprof Care. 2012;27(1):27-33. http://dx.doi.org/10.3109/13561820.2012.715098

34. Baker L, Egan-Lee E, Martimianakis MA, Reeves S. Relationships of power: implications for interprofessional education. J Interprof Care. 2011;25(2):98-104. http://dx.doi.org/10. $3109 / 13561820.2010 .505350$

35. Mandy A, Milton C, Mandy P. Professional stereotyping and interprofessional education. Learn Health Soc Care. 2004;3(3):154-70. http://dx.doi.org/10.1111/j.14736861.2004.00072.x

36. Mickan S, Hoffman SJ, Nasmith L. Collaborative practice in a global health context: Common themes from developed and developing countries. J Interprof Care. 2010;24(5):492-502. http://dx.doi.org/10.3109/13561821003676325

37. Haddad AE, Campos F, Freitas M, Brenelli S, Passarela T, Ribeiro T. Programa de educação pelo trabalho para a saúde-PET-Saúde. Cad ABEM. 2009;5(1):6-12.

38. Abrahão AL, Cordeiro BC, Marques D, Daher DV, Teixeira GHMdC, Monteiro KA et al. A pesquisa como dispositivo para o exercício no PET-Saúde UFF/FMS Niterói. Rev Bras Educ Med. 2011;35(3):435-40. http://dx.doi.org/10.1590/S0100-55022011000300019

Costa MV, Borges FA. El Pro-PET-Salud frente a los retos de la educación profesional en salud. Interface (Botucatu). 2015; 19 Supl 1:753-63.

Este trabajo trata de explorar los principales cambios inducidos por la actual reorientación de las políticas de formación profesional en la salud, en particular el Programa Nacional para la Reorientación de la Formación Profesional y el Programa de Educación en el Trabajo para la Salud - el Pro- PET-Salud. Utilizaron como fuente de datos los reportes de las instituciones y de la asesoría del Ministerio de la Salud, que están disponibles en la plataforma FORMSUS. Fue adoptado el análisis de contenido temático como técnica de análisis de datos. El Pro-PET-Salud está induciendo cambios neurálgicos en educación para la salud: fortalecimiento de la articulación enseñanza/servicio; diversificación de los escenarios de las prácticas y la innovación de los métodos de educación; fomento de la investigación en conjunto con las necesidades sociales y de salud y estimulando la educación interprofesional.

Palabras clave: Educación en salud. Servicios de integración docente assistencial. Políticas públicas. 
